\title{
Structural Modification of PVA Nanofibers by Water Vapor Annealing
}

\author{
Taiyo Yoshioka*1, Yutaka Kawahara ${ }^{* 2}$, Masaki Tsuji ${ }^{* 3}$, and Andreas K. Schaper ${ }^{* 1}$ \\ ${ }^{* 1}$ Material Sciences Center, Philipps University, Hans Meerwein Str., D-35032 Marburg, Germany \\ ${ }^{* 2}$ Department of Biological \& Chemical Engineering, Gunma University, 1-5-1 Tenjin-cho, Kiryu, Gunma \\ 376-8515, Japan \\ ${ }^{* 3}$ Institute for Chemical Research, Kyoto University, Uji, Kyoto 611-0011, Japan
}

\begin{abstract}
The water vapor treatment has been applied to the annealing of as-electrospun partially oriented poly(vinyl alcohol) nanofibers, and it has been found that the fibers can be crystallized without dissolution at a temperature of $23{ }^{\circ} \mathrm{C}$ in a relative humidity of $70 \%$, and left for 3 or 7 days.
\end{abstract}

(Received 7 February, 2011 ; Accepted 22 February, 2011)

\section{Introduction}

Recently significant scientific attention has been paid on nanofibers and/or nanocomposites because of their excellent and peculiar physical properties. In the production of such nano-scaled materials, poly(vinyl alcohol)(PVA) is one of the most attractive candidates due to its water-soluble property and good performance in practical use. A large variety of nanocomposites have been prepared using PVA as a matrix and nanoreinforcements, e.g. silica [1], cellulose whiskers [2], single-walled carbon nanotube (SWNT) [3]. When the electrospinning of PVA/silica composite was made, PVA was changed from semicrystalline to amorphous state because the hydroxyl groups of PVA molecules would react with silica species, resulting in the decreasing of crystallinity without respect to the diameter of PVA/silica hybrid fibers [1]. To the contrary in the case of aselectrospun SWNT/PVA nanocomposite fibers, the crystallization of PVA was enhanced, indicating the occurrence of a SWNT-induced nucleation crystallization of the PVA phase [3]. These data indicate that the crystallinity of PVA matrix tends to be influenced by the surface properties of nanoreinforcements.

The physical properties of nanocomposites will be enhanced by the crystallization of PVA matrix. For this purpose we have adopted the water vapor annealing. Assender and Windle [4] have reported previously that the PVA cast film annealed under humid conditions shows a significantly higher degree of crystallinity. We have also found out the effect of water vapor treatment for the crystallization of as-electrospun silk fibroin nanofibers [5]. However those water vapor treatments have been made under the humid conditions higher than $97 \%$ relative humidity $(\mathrm{RH})$. Such conditions are not applicable to the nanofibers made of water-soluble PVA.

In this paper the water vapor treatment conditions suitable for the crystallization of PVA nanofibers were investigated.

\section{Experimental}

\subsection{Electrospinning}

PVA $(\mathrm{Mw}=66,000-79,200$, saponification value $=$ $96 \mathrm{~mol} \%$ ) was obtained from Wako Pure Chemical Industries Ltd. (Osaka, Japan) and used as received. The distance between the tip of the needle and the collector was $12 \mathrm{~cm}$. An aqueous solution of $6.4 \mathrm{wt} \%$ PVA was used for the electrospinning at a flow rate of the polymer solution and an applied voltage of $0.39 \mathrm{ml} \mathrm{h}^{-1}$ and $16 \mathrm{kV}$, respectively. The photo for the spinning apparatus is shown in Fig. 1. For the convenience of polarizing microscopy, the fibers were collected roughly in parallel fashion each other using a rotation collector shown in Fig. 1. In order to avoid any effects of the collection process on the fiber structure, a slow enough rotation speed of ca. $20 \mathrm{~m} / \mathrm{min}$ was adopted. It is well known that the bending and splaying jet is stretched to higher ratios to allow accumulation of finer fibers with uniform alignment as the collector rotating speed increases [6].

\subsection{Water vapor treatment}

The electrospun fibers, collected between the fins of the electrode, were picked up on the cupper grids for transmission electron microscopy (TEM), moved into a sealed humid-chamber, which was kept at a temperature of $23{ }^{\circ} \mathrm{C}$ in $70( \pm 1) \% \mathrm{RH}$, and left for 3 or 7 days. After 
vapor treatment, the TEM grids were taken out of the chamber, dried in a desiccator for 3days, and followed by the aluminum coating for successive TEM.

\subsection{Characterization}

Crystallographic analysis of single nanofibers was done by selected-area electron diffraction (SAED) in a transmission electron microscope JEM-3010 (JEOL Ltd., Tokyo, Japan) at an accelerating voltage of $300 \mathrm{kV}$ using a slow scan CCD camera (Gatan Inc., Pleasanton, USA) for recording images and SAED patterns. In order to suppress electron charge-up and to support each fragile fiber on the grid, copper grids covered with thin carbon support-film were used. Thin aluminum coating of the specimens was applied for calibrating the diffraction camera length.

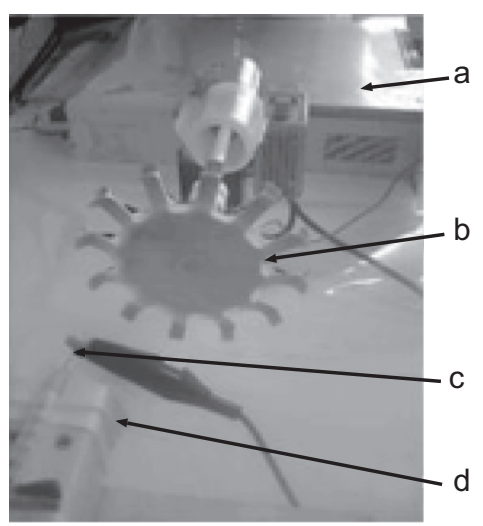

Fig. 1 Electrospinning apparatus: a, power supply unit; b, collector $(-)$; c, needle $(+)$; d, micro-pump.
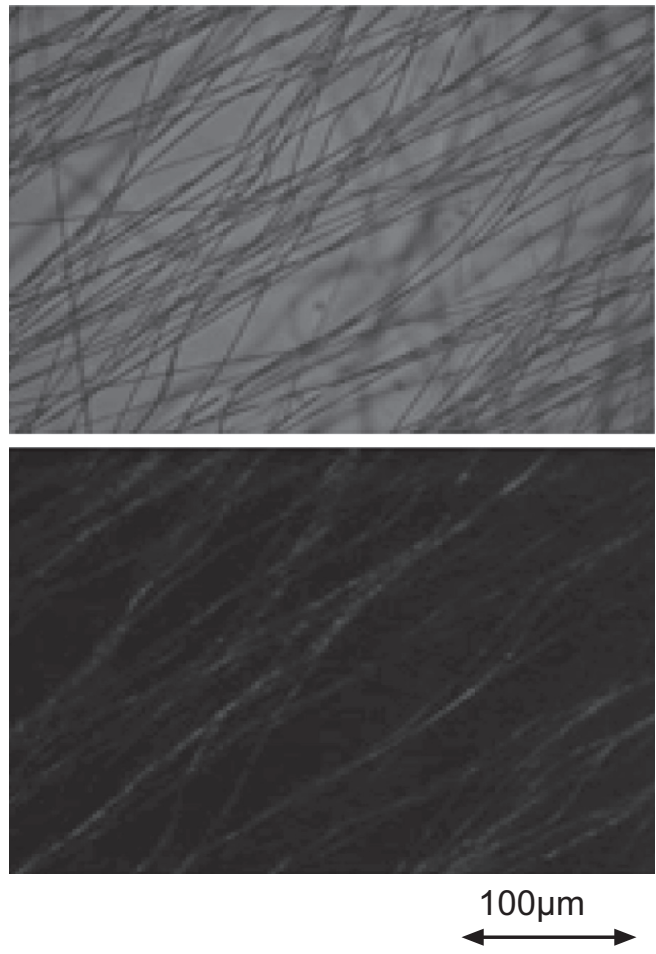

Fig. 2 Polarizing microscopic images captured under : top, one nicol; bottom, crossed nicols.

\section{Results and discussions}

Polarizing microscopic images for the as-electrospun fibers are shown in Fig. 2. Although the brightness and

(a)
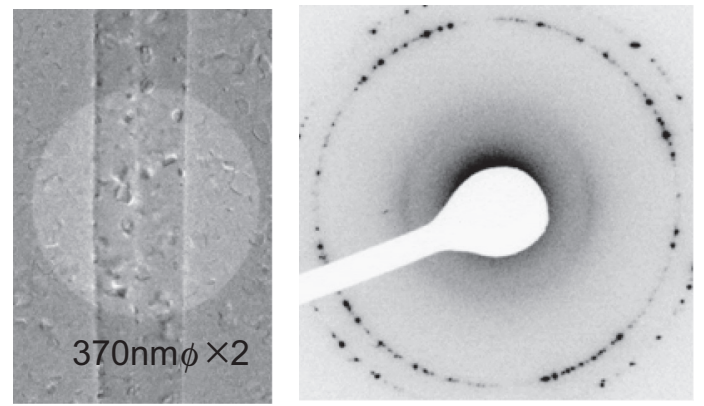

(b)
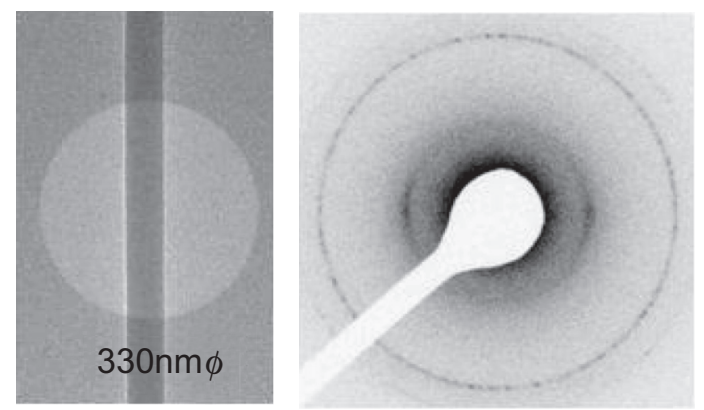

(c-1)
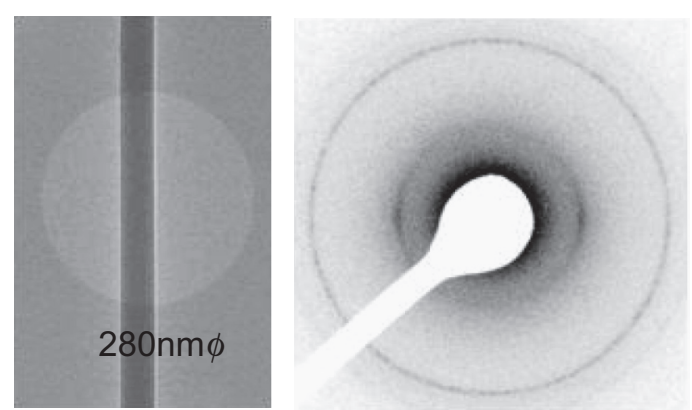

(c-2)
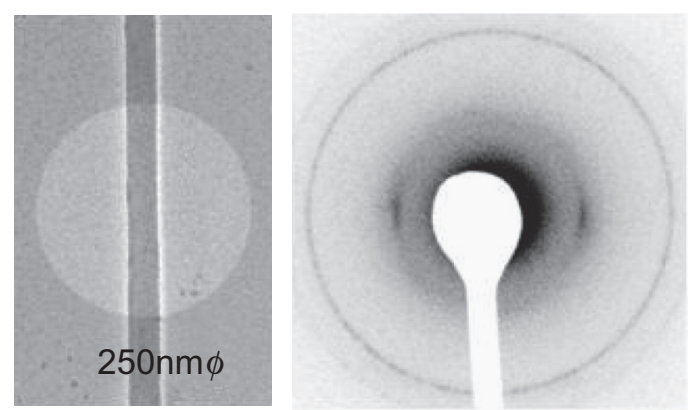

Fig. 3 TEM images and corresponding SAED patterns for the PVA nanofibers: a, control; subjected to the water vapor treatment at $23{ }^{\circ} \mathrm{C}$ and $70 \% \mathrm{RH}$, then left for: b, 3 days ; c-1 and c-2, 1 week. 
the contrast of the images are fairly low, the shape of the fibers can be recognized under crossed nicols position. It is seen that molecular chains of PVA are weakly oriented along the fiber axis.

The influence of water on the crystalline phase of PVA was previously reported by Assender and Windle [4]. They prepared PVA cast films equilibrated to $97 \% \mathrm{RH}$ and made annealing of these films in water vapor at $100^{\circ} \mathrm{C}$. The sample annealed under humid conditions showed a significantly higher degree of crystallinity as compared with one annealed under the dry conditions. In the case of electrospun PVA fibers, however, such severe annealing will bring about the complete dissolution of fibers due to the high surface-tovolume ratio. On the contrary, when the annealing of PVA is performed in dry state, the annealing temperature should be controlled higher than $230^{\circ} \mathrm{C}$ for the successful crystallization. However such high annealing temperature is not applicable to the crystallization of PVA when PVA is used as a matrix of nanocomposites reinforced by hybridization with natural nanoreinforcement such as cellulose whiskers [2]. Therefore the applicability of water vapor treatment to the crystallization of PVA nanofibers has been studied and it has been found that PVA nanofibers can be crystallized at room temperature under humid conditions much lower than $97 \%$ RH. The changes in SAED patterns by the water vapor treatment at $23^{\circ} \mathrm{C}$ in $70 \% \mathrm{RH}$ are shown in Fig.3. In general, nanofibers have a wide distribution in diameter, which means that the draw ratio i.e. molecular orientation differs depending on the diameter of the fiber [7]. Thus it is important to prepare as-electrospun fibers with diameters in the almost same range. In this study as-electrospun fibers of $300 \pm 70 \mathrm{~nm}$ in diameter were used (see Fig. 3). When the partial dissolution of PVA occurred during the water vapor treatment, the fiber diameter became apparently thicker. Without dissolution, no change in diameter was observed because the fibers were completely settled on the cupper grids. SAED peaks of $0.457 \mathrm{~nm}$ on the equator in Fig. 3 are assigned to the overlapping of 101 and 10-1 reflections [4]. In the TEM image for control fibers in Fig. 3(a) the rough surface of the fiber is due to the artifact of aluminum settled. As for control fibers the ED peaks from the crystallites are weak and not so concentrated on the equator, which is consistent with the result obtained from the polarizing microscopic observations (see Fig. 2). From the comparison between Figs. 3(b) and (c) with Fig. 3(a) it is distinct that the SAED peaks on the equator have become stronger and a little concentrated through the water vapor treatment. It is seen that crystal orientation along the fiber axis as well as crystallization of PVA have been enhanced. This tendency was confirmed to have a high reliability through the same analysis applied to more than 30 fibers. It is possible that the electrospun PVA fibers will be able to be fully crystallized if optimal humid conditions are found out and applied to the annealing of the fibers.

\section{Conclusions}

The water vapor treatment was applied to the annealing of as-electrospun partially oriented PVA nanofibers, and the fibers were crystallized at $23^{\circ} \mathrm{C}$ in $70 \% \mathrm{RH}$, left for 3 or 7 days. This treatment will become more useful if the optimal humid conditions are found out and applied to the annealing. The conditions will be reported elsewhere.

\section{References}

1. C. Shao, H. Y. Kim, J. Gong, B. Ding, D. R. Lee, and S. J. Park, Materials Letters, 57, 1579 (2003).

2. M. Roohani, Y. Habibi, N. M. Belgacem, G. Ebrahim, A. N. Karimi, and A. Dufresne, Eur. Polym. J., 44, 2489 (2008).

3. M. Naebe, T. Lin, M. P. Staiger, L. Dai, and X. Wang, Nanotechnology, 19, 305702 (2008).

4. H. E. Assender, A. H. Windle, Polymer, 39, 4295 (1998).

5. Y. Kawahara, A. Nakayama, N. Matsumura, T. Yoshioka, and M. Tsuji, J. Appl. Polym. Sci., 107, 3681 (2008).

6. R. Inai, K. Kotaki, and S. Ramakrishna, Nanotechnology, 16(2), 208 (2005).

7. T. Yoshioka, R. Dersch, M. Tsuji, and A. K. Schaper, Polymer, 51, 2383 (2010). 